\title{
Versatile and scalable pulse compression platform
}

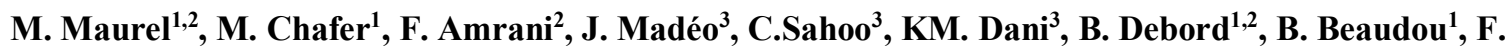 \\ Gérôme ${ }^{1,2}$ and F. Benabid ${ }^{1,2}$ \\ ${ }^{1}$ GLOphotonics SAS, 123 avenue Albert Thomas, 87060 Limoges, France \\ ${ }^{2}$ GPPMM group, XLIM CNRS UMR7252, Université de Limoges, Limoges, France \\ ${ }^{3}$ Femtosecond Spectroscopy Unit, Okinawa Institute of Science and Technology Graduate University, Okinawa 904-0495, Japan \\ Authore-mail address: f.benabid@xlim.fr
}

Abstract: We report on a user-friendly sub-100 fs nonlinear pulse compression platform named

FastLas. The compressor is based on gas fillable inhibited-coupling fibers and can be scaled over a large parameter-space of the input pulse.

\section{Introduction}

OCIS codes: $320.5520,320.7140,060.5295$

Over the last two decades, ultra-short pulse (USP) lasers have witnessed a dramatic progress in their design, performance and in their applications. Nowadays, commercially available table-top lasers emitting pulses with duration of hundreds of femtosecond and milli-Joule energy have become a common place. Among the most representative of these USP lasers, we count Ti:Sapph laser emitting at around $800 \mathrm{~nm}$ for, Yb-based laser emitting around $1 \mu \mathrm{m}$, and Er-based laser emitting around $1.5 \mu \mathrm{m}$, and their frequency-doubled and tripled versions. Within this landscape, the laser pulse-duration is often larger than $100 \mathrm{fs}$, and achieving sub-100 fs pulse duration still remains a challenge. Among the limiting factors in pulse compression is the narrow gain-bandwidth of some lasers (e.g. Yb-based and Er-based lasers), which requires further spectral-broadening followed with a post-compression, and/or nonlinear compression. Furthermore, even with larger gain bandwidth lasers such as Ti:Sapph, complex setup are required for efficient dispersion compensation. Finally, the high sensitivity of current pulse-compression schemes to well defined parameter set of the input laser such as pulse energy, pulse duration, and wavelength, makes it extremely challenging to have efficient and scalable USP compressors. This situation is illustrated by state-of-theart of Yb-based lasers. The latter offers a superior power-scaling, it can be operated in different operational modes, such as emitting from $\mathrm{CW}$ regime to $100 \mathrm{fs}$ pulses with repetition rate from $\mathrm{kHz}$ to $\mathrm{GHz}$ repetition rate. Achieving sub-100 fs remains a challenging task despite the implementation of ingenious schemes to overpass this limitation [1]. In turn, this limits their impact in some of the emerging industrial applications where processing of some materials such as dielectric or semiconductor require $10-100 \mu \mathrm{J}$ level pulses with pulse-width below $300 \mathrm{fs}$.

Here, we exploit the engineerable dispersion, the broadband transmission and high energy handling of InhibtedCoupling guiding hollow-core photonic crystal fiber (IC-HCPCF) to propose a USP compression platform coined FastLas, which operates on energy range between few microjoules to hundred microjoules and efficient for a wide variety of wavelengths.

\section{FastLas}

The FastLas presented in Fig 1 is a table top $(470 \times 288 \times 97 \mathrm{~mm})$ module. Beside including a precise control over vacuum or gas inside the fiber and water cooling extending average power handling, it has been designed to accept the wide variety of inhibited coupling (IC) fiber developed by GLOphotonics covering compression needs for USP laser from UV to mid IR [2].

(a)

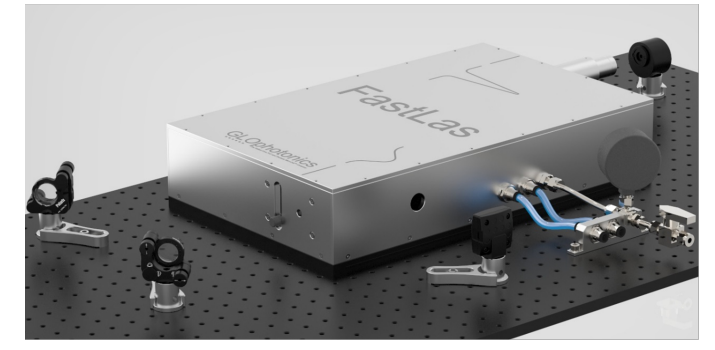

(b)

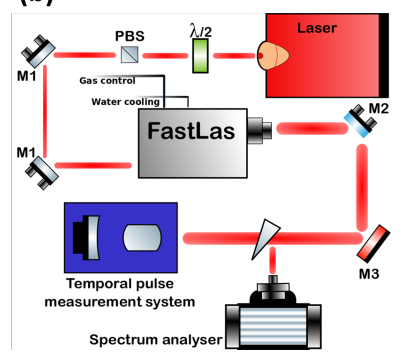

Fig 1. a- Artistic view of the table-top GLOphotonics FastLas. b- Typical set-up for the use of the FastLas.

Figure 1-b represents a typical experiment set-up for the use of FastLas. A typical set-up can be composed by the USP laser to compress, a control of input power done by polarizing optics, and beam-steering mirrors (M1) to couple the beam into the FastLas. At the output, the beam is characterized spectrally and temporally using beamsteering mirrors. The choice of the latter depends on the structure of the broadened spectrum and its chirp. When the spectral broadening is driven by SPM, a post-compression stage is necessary and done thanks to dispersive mirrors 
such as Gires-Tournois Interferometer or Group Delay Dispersion (GDD) mirror to both compensate the dispersion and to steer the beam (M2 and M3 in Fig. 1-b). Given the very low value of the IC-HCPCF dispersion and the subsequent small chirp of the generated SPM spectrum in low pressure inert gases, one GDD mirror with multiple bounces is often sufficient. In the case of self-compression via soliton generation, dispersive optics is not required.

\section{Performances}

In this section we present the performances of different FastLas designed for different input beam (pulse duration and wavelength). To do so, SPM-based postcompression or solitonic-based self-compression mechanisms are used. The first compression demonstration is carried out for a $250 \mathrm{fs}$ UV laser emitting at $343 \mathrm{~nm}$. In this configuration, by filling FastLas with Argon gas at 15 bar the spectrum is broaden by more than $20 \mathrm{~nm}$. Then a post-compression is realized and the pulse duration is compressed down to $50 \mathrm{fs}$, corresponding to a compression factor of 5 (see Fig. 2). The energy contained in the compressed pulse corresponds to $51 \%$ of the total output energy, measured at 1/e.
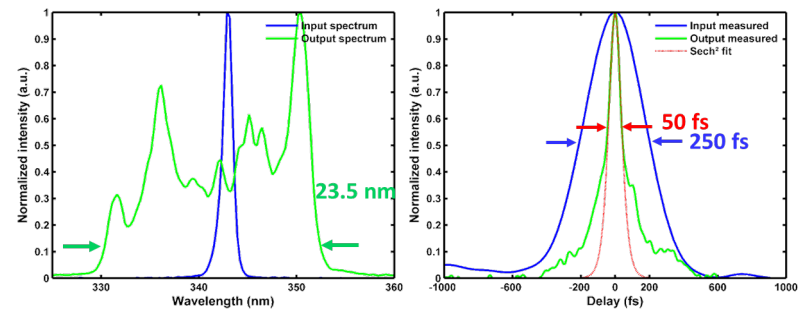

Fig 2. Input (blue), output (green) and fitted (red) pulse in the spectral and temporal domain for the UV FastLas.

A second demonstration of compression has been carried out with a laser emitting at $1030 \mathrm{~nm}, 270 \mathrm{fs}$ and $2 \mathrm{MHz}$. The process of compression is now based on a self-compression. A new HC-PCF and gas configuration has been set to optimize the anomalous dispersion regime. The FastLas filled with 3 bar of Argon is able to compress by a factor 9 the pulse down to $30 \mathrm{fs}$ at $50 \mathrm{~W}$ with an energy efficiency of 55\% (see Fig. 3).
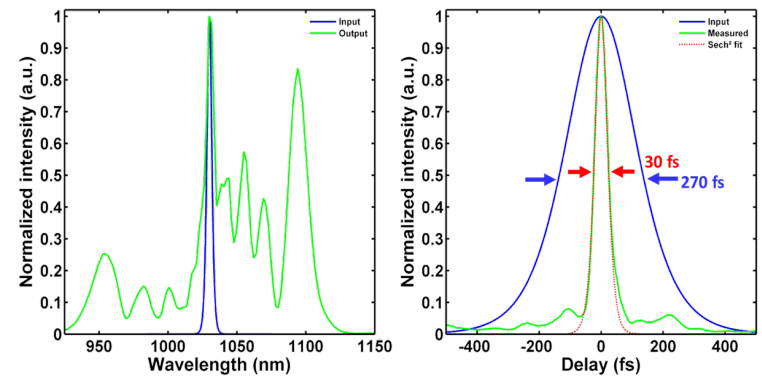

Fig 3. Input (blue), output (green) and fitted (red) pulse in the spectral and temporal domain for the $1 \mu \mathrm{m}$ FastLas at $2 \mathrm{MHz}$.

For a $580 \mathrm{fs}, 1030 \mathrm{~nm}$, input pulse, a third FastLas is presented based on the same self-compression scheme. At $256 \mu \mathrm{J}$, compression down to $22 \mathrm{fs}$ is presented fig 4 , which corresponds to an impressive compression factor of 26 , the strongest reported compression factor using a HC-PCF. The stability of the compression has been investigated and the Fig. 4 shows that the pulse duration is kept constant with a standard deviation of $0.5 \mathrm{fs}$.
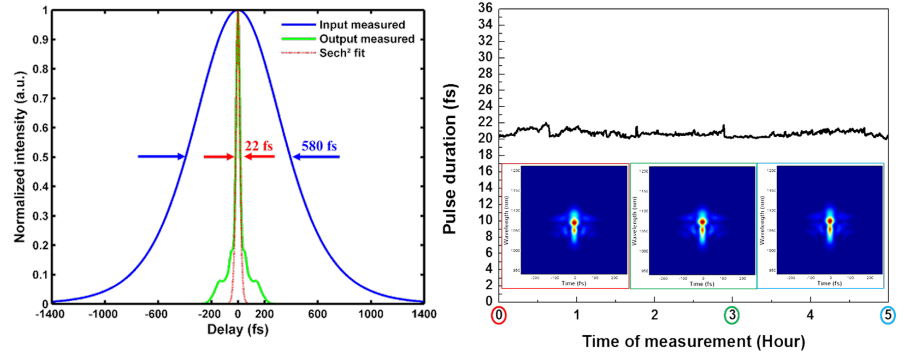

Fig 4. Input (blue), output (green) and fitted (red) pulse in the temporal domain for the $1 \mu \mathrm{m}$ FastLas at $256 \mu \mathrm{J}$. Pulse duration stability over 5 hours, FROG traces are added 3 different moments.

\section{References}

[1] J. Pouysegur et al., "Numerical and experimental analysis of nonlinear regenerative amplifiers overcoming the gain bandwidth limitation,” IEEE J. Sel. Top. Quantum Electron. Inst. Electr. Electron. Eng., vol. 21, no. 1, 2015.

[2] Maurel et al., "Optimized inhibited-coupling Kagome fibers at Yb-Nd:Yag $(8.5 \mathrm{~dB} / \mathrm{km})$ and Ti:Sa (30 dB/km) ranges," Opt. Lett. 43, 1598-1601 (2018) 